\title{
New Questions of Methodology of Scientific Knowledge and History of «Research Programs» (Methodological Approaches of Lakatos, Popper, Kuhn and Duhem)
}

ISSN: 2576-8840

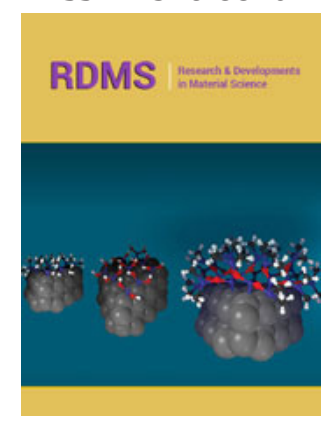

For HTML Version scan this QR code:

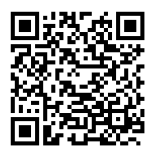

${ }^{* 1}$ Corresponding author: Kochetkov $\mathrm{AV}$, Perm national research polytechnical university, Russia

Submission: 㘹 February 19, 2019

Published: 軘March 01, 2019

Volume 10 - Issue 1

How to cite this article: Kochetkov AV, Fedotov PV. New Questions of Methodology of Scientific Knowledge and History of «Research Programs» (Methodological Approaches of Lakatos, Popper, Kuhn and Dyugem). Res Dev Material Sci. 10(1). RDMS.000730.2019.

DOI: 10.31031/RDMS.2019.10.000730

Copyright@ Kochetkov AV, This article is distributed under the terms of the Creative Commons Attribution 4.0 International License, which permits unrestricted use and redistribution provided that the original author and source are credited.

\author{
Kochetkov AV1* and Fedotov PV ${ }^{2}$ \\ ${ }^{1}$ Perm national research polytechnical university, Russia \\ ${ }^{2}$ Expert of JSC Research Center of Technical Regulation, Russia
}

\begin{abstract}
A comparative analysis of the methodological approaches of different authors is carried out. The advantages and disadvantages of different methods of scientific knowledge are compared. In the article, using the methodological approach, the analysis of the current state of the molecular theory of matter is carried out. Analysis of the modern theory of molecular physics is mainly based on the theory of research programs of I. Lakatos. Since, it is the theory of Lakatos, according to the authors, meets the criteria of the most complete and in-depth analysis in the field of the methodology of scientific knowledge. Instead of the static theory of Lakatos, the authors propose a dynamic concept of "research programs". Such an approach allows us not only to analyze already existing physical theories, but also to develop new ones, taking into account the accumulated problems. Since, based on the analysis, according to Lakatos's method, the modern molecular theory of matter is in a stage of stagnation, the authors propose a new concept of molecular physics. Author's analysis and suggestions for the modernization of the molecular theory were published earlier, so the article does not provide a full analysis and justification of the proposals.
\end{abstract}

Keywords: Science methodology; Theory of research programs; Molecular theory of substance; Development; Education; Scientific theories; Hard core; auxiliary hypotheses

\section{Introduction}

New questions of philosophy teaching in technical universities are devoted to the methodological approaches of Lakatos, Popper, kun and dugem in relation to the dialectics of scientific knowledge [1-8]. It is believed that the theory of "Lacatos research programs" show only the development of scientific theories, but this is not true. In fact, the Lacatos theory reflects the full life cycle of scientific theories. In the initial period of development and formation of scientific theory genius insight plays a progressive role in science. But then, passing through the peak of "public good", the outdated paradigm (according to Kun) or the research program (according to Lakatos) begins to play the opposite role. So lacatos characterizes the evolution of Bohr's theory with the words: "thus, the rational position in relation to the 'grafted' programs is to use their heuristic potential, but not to put up with the chaos in the bases from which they grow. The" old " (until 1925) quantum theory was mainly subject to such a setting. After 1925 the "new" quantum theory switched to "the anarchist position", and modern quantum physics in its "Copenhagen" interpretation has become one of the main strongholds of philosophical obscurantism" [6, p. 101].

All the statements about the quantum theory can be said with no less success about the modern molecular theory. As lacatos wrote: "this means that being under the impression of a long period of empirical success of this theory, scientists can decide that to refute this theory is generally unacceptable. In accordance with this decision, the scientists try to eliminate 
obvious anomalies (or not trying to do it) by means of auxiliary hypotheses or other "conventionalists above" [6, C. 33]. Simply put: the long success of the theory leads to the idea that the theory is correct because for too long no one could refute it, and if this could not be done in the past, then it will not be possible to do in the future, and the older the theory, so it is true, according to the supporters of the theory. However, as the history of science shows - it never works. For some reason, everyone forgets that Aristotle's physics was considered the truth of one and a half thousand years, but this did not save her from refutation. So, the question is not whether any theory can or cannot be disproved, the question is when it will happen and how. The main question is how the outdated theory will be replaced, whether the new theory will be better than the old one. "A scientific theory is declared invalid only if the alternative is suitable to take its place. There is not a single process revealed by the study of the history of scientific development, which in General would resemble the methodological stereotype of refuting the theory through its direct comparison with nature. This statement does not mean that scientists do not abandon scientific theories or that experience, and experiment are not important for such a refutation process. But that means (ultimately, this will be the Central point) that sentencing a scientist to abandon a previously accepted theory is always based on something more than comparing theory to the world around us. The decision to abandon a paradigm is always simultaneously the decision to take another paradigm, and the sentence leading to this decision include, as a comparison of both paradigms with nature and the comparison of paradigms with each other" [5, C. 110].

Based on these principles, the authors propose a new "research program" in relation to molecular theory.

The meaning of the new "program" is that all these inconsistencies of the modern molecular theory can be easily removed within the molecular-photon hypothesis expressed by the authors [3]. The reasons for the frequent use of the concept of lacatos research programs are that He described the most complete concept of scientific knowledge. Neither Popper, nor Kuhn, nor Duhem could describe the full picture of scientific knowledge. So, Popper was the most idealistic of all idealists. According to the ideas of Popper, it is the author of a scientific theory should be first and foremost, the gravedigger of their own offspring. "The new theory, like all unconverted theories, may be false. Therefore, the theorist struggles to find false theories among a multitude of unproven competitors; he tries to" catch " them. In other words, he seeks against each the unbroken theory to think of cases or situations in which, if it is false, its falsity could occur. Thus, he will try to plan rigorous tests and decisive verification situations. In fact, this means the construction of a falsifying law, that is, a law whose level of universality can be so low that it will not be able to explain the success of the theory to be tested, but which, nevertheless, can suggest a decisive experiment-an experiment that can refute, depending on its outcome, or a theory that is subject to verification, or falsifying its theory" [7, p.18]. Thus, the idea of Popper the author of the theory needs constantly, until the refutation of their own theories seeks to refute their own theories. Such an idea, in principle, can allow the author of the theory not to stop there, but to constantly look for the possibility of refuting his own theory, in order to build an even more true theory. Ideally, the method is very good, but in practice rarely implemented. "The original idea of K. Popper arose as a result of thinking through the consequences arising from the collapse of the most supported scientific theory of all time: mechanics and gravitation theory of I. Newton. K. Popper concluded that the valor of the mind is not to be careful and avoid mistakes, but to uncompromisingly eliminate them. To be bold, putting forward hypotheses, and merciless, denying them the motto of Popper" [6, p. 8]. "The honor of intelligence is not defended in the trenches of evidence or 'verifications' surrounding someone's position, but by the precise definition of the conditions under which that position is deemed unfit for defense. Faith human nature and therefore forgivable weakness, it is necessary to keep under control criticism; but the bias (commitment), says Popper, is a grave crime intelligence" [6, p. 9].

Naturally, the basis of Popper's ideas is the concept of "scientific honesty". "Scientific honesty requires to constantly strive for such an experiment that in case of contradiction between its result and the theory being tested, the latter was discarded. Falsificationist requires that refuted the statement unconditionally rejected without equivocation" [6, p. 17]. Popper lacatos's main criticism is expressed in the words:" and that is to say, even in natural science, the recognition of the theory depends on the quantitative superiority of its supporters, the strength of their faith and vocal cords, what remains to social Sciences; so, the truth is based on strength " $[6, \mathrm{p} .10]$. Under these conditions, it is difficult to hope that the authors and followers of the theory will seek the truth, perceiving "their" theory not as the ultimate truth, but as an intermediate (next) step in the knowledge of the truth. At the same time, it is uncompromising to eliminate the errors of "one's" theory. Unlike Popper, the main idea of Kuhn is the concept of "normal science". Here is how himself kun defined this the notion of: "In this essay the term" normal science " means study, firmly based on one thing or several past scientific achievements-achievements, which in for some time admit certain scientific community as basis for his further practical activities. Today, such achievements are set out, albeit rarely in their original form, in elementary or advanced textbooks. These tutorials explain the nature of accepted theory, illustrate many or all of its successful applications, and compare these applications with typical observations and experiments" [5, p. 28].

Further Kuhn notes: "The purpose of normal science in no way requires the prediction of new types of phenomena: phenomena that do not fit in this box, often, in fact, generally overlooked. Scientists in line with normal science do not set themselves the goal of creating new theories, they are usually intolerant to the creation of such theories by others [1]. On the contrary, research in normal science is aimed at the development of those phenomena and theories, the existence of which the paradigm obviously assumes" [5, p. 45]. Noticing thus: "it is Possible that it should be referred to number of shortcomings" [5, p.45]. Note the fundamental difference between Kuhn's and Popper. Kun notes that at a certain stage, the scientific community takes one of the scientific paradigms for Truth and then does not look for grounds for its falsification, but, on the 
contrary, protects the accepted paradigm. Popper also believes that as soon as the scientist finishes developing a new theory, he should immediately start looking for an opportunity to refute it. Lakatos criticizes Thomas Kuhn because of his glorification of normal science. "The scientist should not agree that the research program turns into Weltanschauung, a kind of embodiment of scientific rigor, claiming to be an all-knowing arbiter, determining what can and cannot be considered a scientific explanation, just as, referring to mathematical rigor, trying to decide what can and cannot be considered a mathematical proof. Unfortunately, it is in this position is T. kun. What he calls "normal science" is really nothing more than a research program that has taken over a monopoly. In fact, research programs use a complete monopoly very rarely, moreover, very briefly, regardless of what efforts are made by the Cartesians, supporters of the Newtonian system or Bohr.. The history of science was and will be the history of rivalry of research programs (or, if you like, "paradigms"), but it was not and should not be an alternation of periods of normal science, the faster the rivalry begins, the better for progress "Theoretical pluralism" better than "theoretical monism" here I agree with Popper and Feuerbend and disagree with Kuhn" [6, p.117].

There is clearly Lakatos comes down to the concept of Popper's scientific integrity. Since, it is difficult to imagine that" the research program that has seized the monopoly "itself will abandon the monopoly and from the state of" the only true "voluntarily move into the category of "equal many". But Lakatos is clearly mistaken, the capture of a monopoly in science, the phenomenon is not as rare as we would like. Of course, if you look at the history of science in retrospect, Lakatos is right, after all, every theory that has captured a monopoly turns out to be refuted and rejected and replaced by a new theory. But sometimes the power of monopoly on truth lasts for several centuries. Kuhn "answers": "as long as the means represented by the paradigm can successfully solve the problems generated by it, science advances most successfully and penetrates to the deepest level of phenomena, confidently using these means. The reason is clear. As in production, in science the change of tools is an extreme measure, which is resorted to only in case of actual need " [5, p. 109]. "In addition, there is a second reason to doubt that the scientist refuses paradigms due to a collision with anomalies or counterexamples... in its pure form, they cannot refute this philosophical theory, for its defenders will do what we have already seen in the activities of scientists when they fought with the anomaly. They will invent countless interpretations and modifications of their ad hoc theories in order to eliminate the apparent contradiction. Many of the relevant modifications and qualifications are actually already in the literature" [5, p. 111]. "Phenomena that do not fit in this box are often, in fact, generally overlooked"[5]. Pierre Duhem believed that the main trouble of physical theories-is the bias of scientists, including experimenters, he wrote, citing Claude Bernard: "People who feed too much confidence in their theories or their thoughts, not only are little able to make discoveries, but they are very poorly observed. They make their observations always with some biased idea and otherwise cannot observe. Producing any experience, they want to see in the results of it only one thing- confirmation of his theory. Therefore, they distort the observation and often neglect the facts very important, only because these facts do not correspond to their purpose. But it is also very natural that people who believe too much in their theories do not believe in others. Such people look at other people from top to bottom and are busy with only one thought-to find mistakes and contradictions in the theories of others. But it is equally bad for science. They make their experiments only to destroy some theory, not to find the truth. They make poor observations, for they enter into the results of their experiments only what agrees with their goal, which they set themselves, omitting everything that is with her in any connection, and carefully eliminating everything that agrees with the idea, which they dispute. So, two opposite paths they come to the same result - juggling science and facts" [2, p. 216].

Appeal Duhem in the sense of Popper offers: "The Conclusion from all this that, before the data of experience need to silence not only someone else's opinion but your own; what you need to accept the results of experience as they are with all the emergency parties and all contingencies" [2, p. 217]. Thus, Kuhn and Lakatos agree that to abandon accepted on faith paradigms (Kuhn) or research program (Lakatos) scientists did not hurry. However, sometimes they do, as Kuhn notes, not only and not so much under the pressure of objective facts, new experiments, but more under the pressure of the convenience of the new theory. The convenience of the new theory is usually that it is more compact, i.e., the postulates of the new theory are formulated in such a way that it is not required, as Lakatos put it, "conventionalist tricks" to explain more facts than it was done within the framework of the old theory. Duhem explains this phenomenon's psychological thinking, but also agrees that a similar phenomenon occurs in science. Popper, on the contrary, believes that scientists should not only immediately abandon the outdated theory, as soon as there is at least one fact that does not fit with the already developed theory, but should (in the idea of Popper) also actively seek a reason to refute the existing theory. Because only in the refutation (falsification) of the existing theory there is a sense of further development of science. The following conclusion suggests that Kuhn and Lakatos describe the actual state of science, in other words "real science", and Popper describes" ideal science", what it should be, if all scientists without exception will strictly follow the principle of" honesty in science " in Popper's terminology. The fundamental difference between Kuhn and Lakatos is that Kuhn adopted in science by a majority of votes of the scientists theory or "achievements, which for some time recognized the particular scientific community as a basis for its further practical activities" [5, p. 28] called "normal science", a term that according to the ideas of Kuhn, were United by the initial postulates and the changes made by scientists under the pressure of new circumstances, which include mostly new experimental data, etc. As kun wrote: "in our days such achievements are stated, though rarely in their original form" [5, p. 29]. Recognizing that more often the foundations of scientific theory in modern textbooks set out not quite as they were originally formulated. To present the foundations of the theory can be different. But if the initial postulates change when the exposition changes, it will be a new theory. 
What Kuhn says should in no way affect the very essence of the basic theory expressed in the initial postulates. However, Kuhn doesn't say a word about it. Lakatos, on the other hand, reflects this point in the construction of the theory, which he calls the "research program". Lakatos notes that any research program consists of a hard (immutable) core and a soft shell. According to the idea of Lakatos, the hard core consists of the postulates of the theory, which cannot be changed. A soft shell includes everything that can change within the framework of the accepted paradigm, without affecting the very foundations of the theory. It is in the soft shell and includes all the "conventionalist tricks", which according to Lacatos inevitably appear as the development of research programs. In fact, based on the General philosophical principle of the infinity of knowledge, any theory cannot be final, so any theory is limited and as the development of knowledge inevitably reveals facts that do not fit into the original postulates of any theory. Because according to Kuhn and Lakatos do not lead to immediate failure of scholars accepted on faith the theory, because scientists have a variety of means to resolve the situation. Kuhn expresses this with the words: "they will invent countless interpretations and modifications of their ad hoc theories in order to eliminate the apparent contradiction. Many of the relevant modifications and qualifications are actually already in the literature" [5, p. 111]. At the same time, kun notes that in modern textbooks not all the facts known to science are given, but only those that confirm the accepted paradigm. Lakatos calls this "conventionalism".

An example of improving the soft shell: The essence of the innovations proposed by the authors of the improved theory of molecular physics can be expressed in several words: in molecular physics it is necessary to consider the interaction of atoms and thermal photons. And the presence of thermal photons in an array of molecules, for example, in a gas, no one denies. Also, there is usually no doubt about the interaction of atoms and photons, including mechanical interaction. But, according to modern theories, this fact is carried in different theories. So, in molecular physics is recognized only mechanical interactions exclusively between molecules, and the interaction between atoms and photons made in the framework of atomic physics and the special theory of relativity. All the main provisions of the claimed research program of the authors were published in the form of a series of articles in peer-reviewed journals [9-21]. In the first of them were collected and analyzed the main provisions of the apologists of the conservative theory. In the following gradually developed the basic ideas of the authors on the construction of a new theory.

\section{Discussion of results}

In the General case for the development of new theories necessary to solve the following scientific tasks:

A. To consider carefully all modern theory.

B. To analyze what constitutes the core and what is included in the shell of modern theory. Which is not an easy task, because in modern scientific and educational literature often the basic postulates of the theory and interpretation, based on additional postulates (not included in the list of basic), mentioned mixed without division into basic (fundamental) and additional.

C. Find the inevitable kernel errors in the form of direct inconsistencies of experimentally established facts and postulates of the kernel of modern theory. As well as inconsistencies in the core and shell of modern theory. Which are defined as places of core inconsistencies (basic postulates) and insufficiently logical interpretations and additional postulates (in a soft shell), which appear as new facts, unknown at the initial period of formulation of the initial postulates. Nevertheless, requiring their inclusion in the established theory (paradigm), for its self-preservation. Exactly what Lakatos called "conventionalist tricks", and Poincare was called "finger dexterity".

D. Construct new kernel postulates to remove inconsistencies, tricks and defaults of the previous program as much as possible. Naturally, this increases the core, because in addition to the initial postulates of the previous theory, proved correct, introduced new postulates, allowing the new theory to explain the facts, which in the old theory required various manipulations and tricks.

\section{Summary}

In our view, the understanding and division of the theory into a solid (immutable) core and a soft shell (consisting of interpretations, modifications, additional hypotheses and theorems, as well as the so-called "conventionalist tricks") constitute an exceptional achievement of the theory of Lakatos research programs. With the emergence of any new theory quite naturally raises a lot of questions. But most of the issues are not related to the shortcomings as such, but to the unusual interpretation of already established opinions and judgments. We are not afraid of criticisms, which we welcome, but against the "butchering of small things", which boil down to discussing minor and often irrelevant issues. This is noted by all psychologists and scientists involved in the history and methodology of science. It is not so difficult for scientists to adopt new attitudes as it is difficult to abandon old and familiar ones [4]. "Such conservative conventionalism has, however, the drawback that does not allow to be released from the prisons we have built, when the initial period of trial and error has already passed, and great decisions have been made. The problem of eliminating theories that have triumphed for a long time is thus not solved. According to the conservative conventionalism, in experiments lacking the strength to overthrow the young theory, but with old, well-established, it is no longer held: this means that as soon as the growing science, the strength of the empirical arguments is reduced" [6, C. 33].

In the development of the static scheme of the Lakatos research platform, the authors state that it is considered in dynamics as a General representation of the phase States of interaction between the nucleus and the soft shell. The fact that Lakatos did not state when and how the research platform, there are various conventionalists tricks. But it's clearly visible in Kuhn, who says 
that the various tricks appear when you reveal facts that do not fit into the framework of the initial postulates but require explanation in the framework of the theory for its self-preservation. Such manipulations require the introduction of additional postulates into the theory, sometimes in the form of hypotheses. Here lies the fine line between the development of the theory and its falsification. The differences are that the development of the theory assumes the nomination of additional hypotheses strictly corresponding to the initial postulates, and falsification is obtained in the form of omissions or unclear formulations to hide the fact that additional hypotheses contradict the initial postulates.

\section{References}

1. Barber B (1961) Resistance by scientists to scientific discovery. Science 134(3479): 596-602.

2. Duhem P (2007) Physical theory: Its purpose and structure. p. 328.

3. Kochetkov, Fedotov PV (2016) Interpretation of experimental data on compressibility of gases under different conditions. Atom-photon gas. Internet-Journal Science of SCIENCE 8(2): 1-12.

4. (2015) Problems of harmonization of radical contradictions in the axiomatics of natural sciences. Mechanical Engineering, p. 320.

5. Kuhn T (1977) The structure of scientific revolutions.

6. Lakatos I (1995) Falsification and the methodology of scientific research programmes. p. 235.

7. Popper KR (2002) Objective knowledge. Evolutionary approach, p. 384.

8. Kovalev SM, Panov VG (1983) Philosophical encyclopaedic dictionary. Academy of sciences of the USSR, LF I'ichev, Acad USSR academy of Sciences Fedoseev, p. 840.

9. Kochetkov AV, Fedotov PV (2016) Heterogeneous phases of water.
Internet Journal Science of SCIENCE 8(5).

10. Kochetkov AV, Fedotov PV (2016) Interpretation of experimental data on compressibility of gases under different conditions atom-photon gas. Internet Journal Science of SCIENCE 8(2).

11. Fedotov PV, Kochetkov AV (2017) Molecular kinetically-potential model of an ideal gas. Internet-Journal Science of SCIENCE 9(3).

12. Kochetkov AV, Fedotov PV (2016) A necessary complement to the theory of real gases. Internet Journal Science of SCIENCE 8(2).

13. Kochetkov AV, Fedotov PV. On the radiation of atoms and molecules technical regulation in transport construction. Electronic Scientific Journal.

14. Kochetkov AV, Fedotov PV (2015) On the different meanings of the concept of entropy. Internet Journal science of SCIENCE 7(6).

15. Kochetkov AV, Fedotov PV (2017) Complete classification of models of ideal gas. Internet Journal Science of SCIENCE 9(5).

16. Kochetkov AV, Fedotov PV (201) Extension of the concept of ideal gas. Internet Journal science of SCIENCE 9(4).

17. Fedotov PV, Kochetkov AV (2016) Thermal photonics on phase transitions of first and second kind. Internet Journal science of SCIENCE $8(5)$.

18. Kochetkov AV, Fedotov PV (2017) Equation of state of gas and the model of an ideal gas. Internet Journal Science of SCIENCE 9(3).

19. Fedotov PV, kochet (2017) Equations of state in the molecular-photon theory of gas. Internet Journal of Science 9(5).

20. Kochetkov AV, Fedotov PV (2016) Clarification of the list of basic parameters of thermodynamic state of gas. Internet-Journal Science of SCIENCE 8(2).

21. Kochetkov AV, Fedotov PV (2016) Phase diagram of water. Internet Journal Science of SCIENCE 8(4). 